\title{
Mass Effect
}

National Cancer Institute

\section{Source}

National Cancer Institute. Mass Effect. NCI Thesaurus. Code C125178.

The pressure and/or displacement of adjacent tissues that results from the growth of a mass. 